\title{
Mycotic aneurysm formation after bacillus Calmette-Guérin instillation for recurrent bladder cancer
}

\author{
Sagar Rohailla MD MSc, Abhijat Kitchlu MD, Mark Wheatcroft MD MBChB, Fahad Razak MD MSc
}

Cite as: CMAJ 2018 April 16;190:E467-71. doi: 10.1503/cmaj.171214

A 61-year-old man was admitted to hospital after a 10-month history of progressive low back pain, functional decline and weight loss. His medical history included nonmuscle invasive bladder carcinoma (stage Ta, highgrade disease) that had been diagnosed three years earlier and had been treated with transurethral resection, followed by intravesical bacillus Calmette-Guérin (BCG) induction therapy for six weeks. The patient was given a dose of one-third of a vial of Connaught ${ }^{\mathrm{T}}-$-derived BCG $(60 \mathrm{mg})$ on each occasion. There had been no traumatic catheterization or concerns about concurrent infection with any of the treatments. The patient had seven episodes of tumour recurrence ( $\mathrm{Ta}$, low-grade disease) that required resection and adjuvant maintenance BCG therapy (three doses at six-month intervals), which he tolerated with no complications. The most recent instillation had been completed three months before the patient's admission to hospital (Figure 1).

The patient's medical history also included coronary artery disease with bypass grafting, hypertension, dyslipidemia and type 2 diabetes. Eleven months before his admission to hospital, he had elected to undergo open repair of a $5.8-\mathrm{cm}$ infrarenal abdominal aortic aneurysm. The patient was a former smoker and did not drink alcohol.

Four weeks before his current admission, the patient had experienced renal impairment, with a creatinine of $186 \mu \mathrm{mol} / \mathrm{L}$ (estimated glomerular filtration rate [GFR] $32 \mathrm{~mL} / \mathrm{min}$ per $1.73 \mathrm{~m}^{2}$ ) and evidence of microalbuminuria (albumin-creatinine ratio $20 \mathrm{mg} / \mathrm{mmol}$ ). In addition, the patient had reported worsening low back pain and an unintentional weight loss of 20 pounds $(9.1 \mathrm{~kg})$. He had no rash, joint pain or other systemic symptoms. After renal ultrasonography and baseline laboratory investigations had shown normal results, the patient underwent a kidney biopsy that showed interstitial nephritis with noncaseating granulomas (Figure 2).

After possible offending medications, autoimmune causes such as lupus nephritis, Sjögren syndrome and sarcoidosis (excluded because of the absence of clinical features and negative antinuclear antibodies, anti-DNA and antinuclear cytoplasmic antibodies) and malignant causes such as multiple myeloma (negative serum-urine protein electrophoresis) had

\section{KEY POINTS}

- Intravesical therapy involving bacillus Calmette-Guérin (BCG) is standard of care for high-grade, noninvasive urothelial carcinoma, with reported cure rates higher than $70 \%$.

- Systemic infection occurs in less than $5 \%$ of patients and likely involves hematogenous spread from the instillation site, promoted by local cystitis, traumatic catheterization or a bleeding site in the bladder.

- Infection of an aortic aneurysm is uncommon, but should be considered when patients present with back pain after BCG therapy.

been excluded, the differential diagnosis had focused on infectious causes, including tuberculous and non-tuberculous mycobacterium. The patient's risk factors for tuberculosis included previously treated latent disease in his ex-wife, although he had reported a negative tuberculin sensitivity test within the previous year. Urine acid-fast bacilli testing and polymerase chain reaction (PCR) testing had been positive for Mycobacterium bovis. Acute interstitial nephritis secondary to disseminated infection after BCG instillation had been diagnosed, and the patient had been given isoniazid $(5 \mathrm{mg} / \mathrm{kg}$ per day, orally), rifampin (10 mg/kg per day, orally), ethambutol $(15 \mathrm{mg} / \mathrm{kg}$ per day, orally) and moxifloxacin $(400 \mathrm{mg} / \mathrm{d}$, orally) for a planned duration of two months, followed by isoniazid and rifampin alone for an additional seven months.

During this subsequent admission, the patient complained of severe low back pain, fatigue and an inability to manage activities of daily living at home. On clinical examination, he looked unwell and cachetic. He weighed $68 \mathrm{~kg}$, compared with his previous weight of $73 \mathrm{~kg}$ one month earlier when the interstitial nephritis had been diagnosed. His blood pressure was $88 / 52 \mathrm{~mm} \mathrm{Hg}$, and his heart rate was 90 beats/min. He was afebrile, but he exhibited point tenderness throughout the lumbar spine and was having difficulty moving because of the severity of his back pain. His abdomen was nontender without obvious pulsation. Distal pulses were palpable in his extremities. The laboratory investigations we performed on admission are summarized in Box 1. 
We used magnetic resonance imaging (MRI) with gadolinium enhancement to rule out discitis or bony involvement as a complication of mycobacterial infection (Pott disease), which showed no evidence of vertebral or epidural disease. However, further review of the MRI suggested possible abnormalities in the abdominal aorta. Subsequent computed tomographic (CT) angiography of the abdominal vasculature showed a wide-mouth pseudoaneurysm that involved the posterior wall of the abdominal aorta at the level of the celiac axis and superior mesenteric arteries (Figure 3). The sac measured $6.7 \times 5.0 \times 3.7 \mathrm{~cm}$, with a superiorly irregularly shaped nonenhancing ring that suggested an ulcerated mural thrombus abutting the lumbar spine. The previously repaired infrarenal tube graft appeared uninvolved. There was no evidence of contrast extravasation or rupture.

We consulted the vascular surgery team who thought that the patient's history and the features seen on CT angiography were consistent with a symptomatic mycotic aneurysm. The patient underwent immediate surgery for patch repair that involved a thoracoabdominal retroperitoneal approach. The aneurysm was repaired successfully. At six months' follow-up, the patient

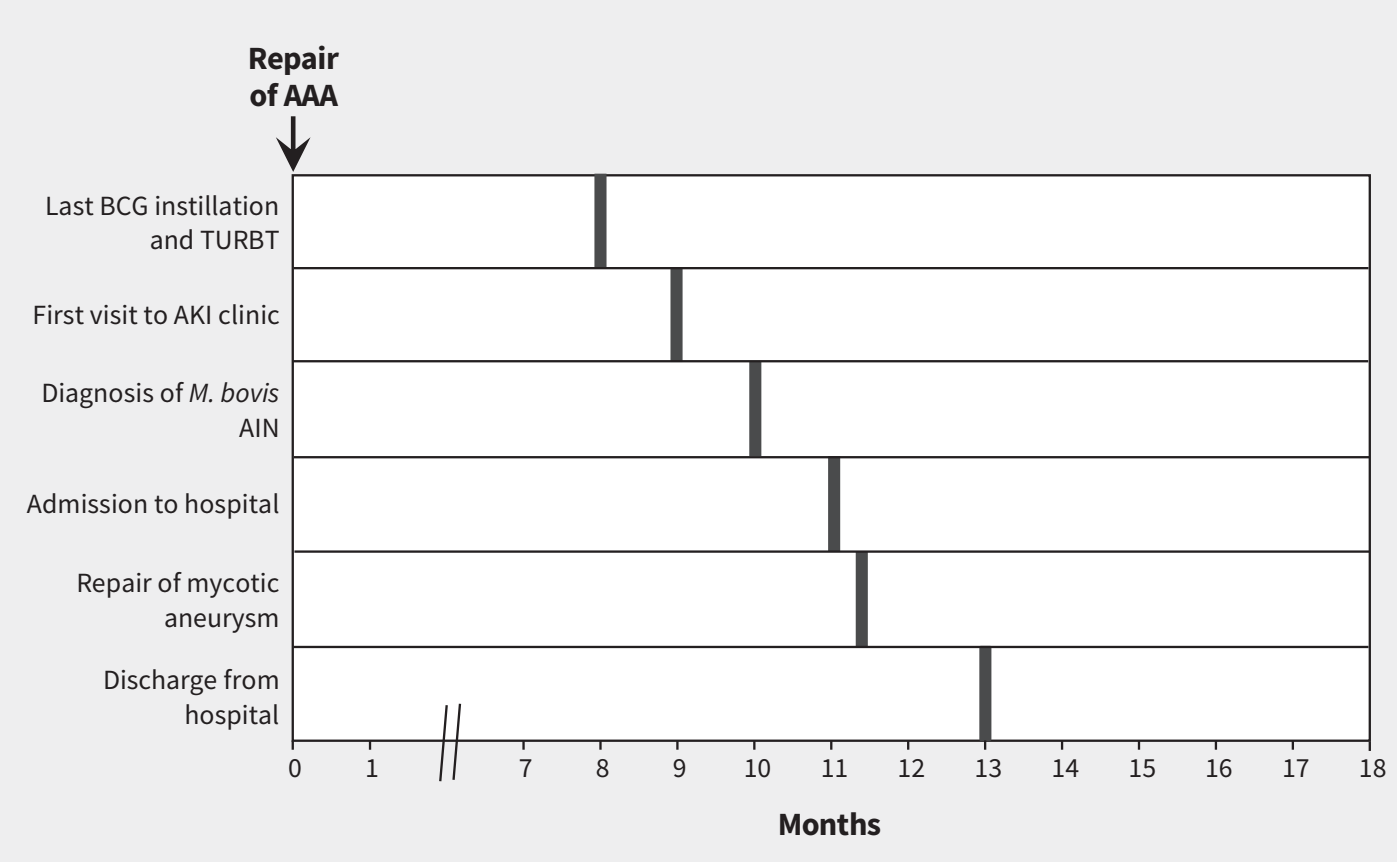

Figure 1: Timeline of events leading up to the admission of a 61-year-old man presenting with back pain, weight loss and functional decline. Bold lines show event onset. Note: $\mathrm{AAA}$ = abdominal aortic aneurysm, AIN = acute interstitial nephritis, AKI = acute kidney injury; $B C G=$ bacillus Calmette-Guérin, M. Bovis = Mycobacterium bovis, TURBT = transurethral resection of bladder tumour.

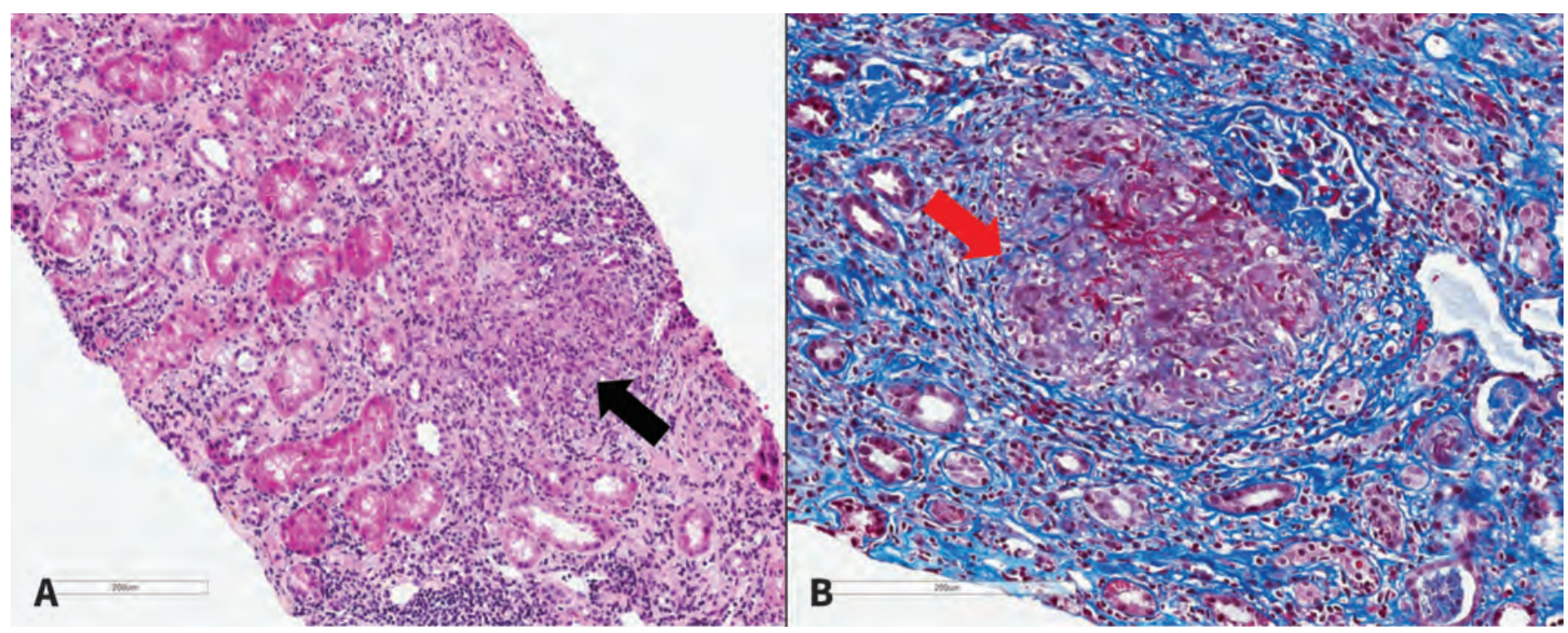

Figure 2: (A) Hematoxylin phloxine saffron stain of a sample from the patient's renal cortex showing nonnecrotizing granulomatous inflammation (black arrow, original magnification $\times 12$ ). (B) Trichrome stain of the renal cortex showing nonnecrotizing granulomatous inflammation (red arrow) (original magnification $\times 15$ ). 
remains well. Postoperative tissue cultures of the aneurysmal tissue were positive for $M$. bovis with +3 acid-fast bacilli (10-90 cells/field) seen on initial smear. Blood cultures that had been drawn after two weeks of anti-tuberculous therapy at the time of admission were negative after six weeks' growth. The patient's creatinine one month after surgery had returned to $98 \mu \mathrm{mol} / \mathrm{L}$. He continues to take antimycobacterial therapy. Followup CT angiography (Appendix 1, available at www.cmaj.ca/lookup/ suppl/doi:10.1503/cmaj.171214/-/DC1) shows an intact repair.

\section{Discussion}

Nonmuscle invasive bladder cancer is the fifth most common cancer in Canada, with about 7800 cases per year. ${ }^{1}$ Most bladder cancers are of urothelial carcinoma origin for which transurethral resection of the tumour is first-line treatment. ${ }^{1}$ Although nonmuscle invasive bladder cancers are associated with five-year survival rates of about $90 \%$, tumour recurrence rates are about $70 \%$, with $30 \%$ of cancers progressing to more advanced stages and grades. ${ }^{2}$ Adjuvant intravesical therapy with live attenuated BCG was developed in the 1970s, and has been shown to reduce recurrence and progression. ${ }^{2,3}$ Bacillus Calmette-Guérin immunotherapy is now considered a mainstay of therapy for highgrade $\mathrm{T} 1$, carcinoma in situ and recurrent Ta tumours (grade A recommendation in the 2017 Canadian Urological Society guideline), with reported cure rates higher than $70 \% .^{1,2}$

The proposed mechanism for immunotherapy involves activation of the patient's immune response to the uptake of attenuated bacterium to produce a generalized inflammatory reaction that then targets cancer cells. ${ }^{4}$ Given the requirement for an intact immune response to mount an antitumour effect, BCG therapy is contraindicated for patients with immuno-suppression. ${ }^{4}$

The profile of adverse effects related to BCG therapy is well documented and includes localized inflammatory changes and

Box 1: Laboratory investigations performed during the patient's admission to hospital and on two occasions preceding admission

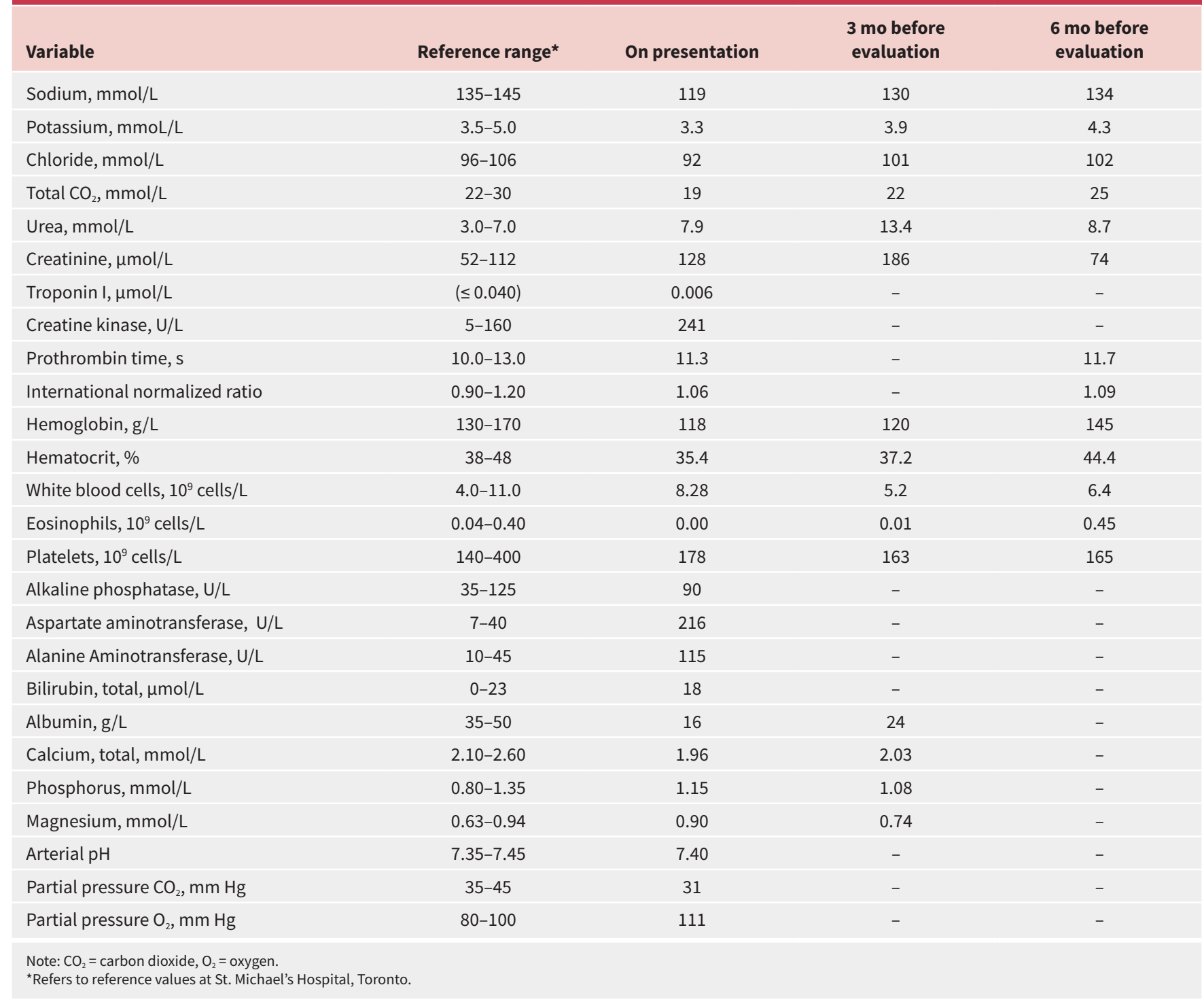



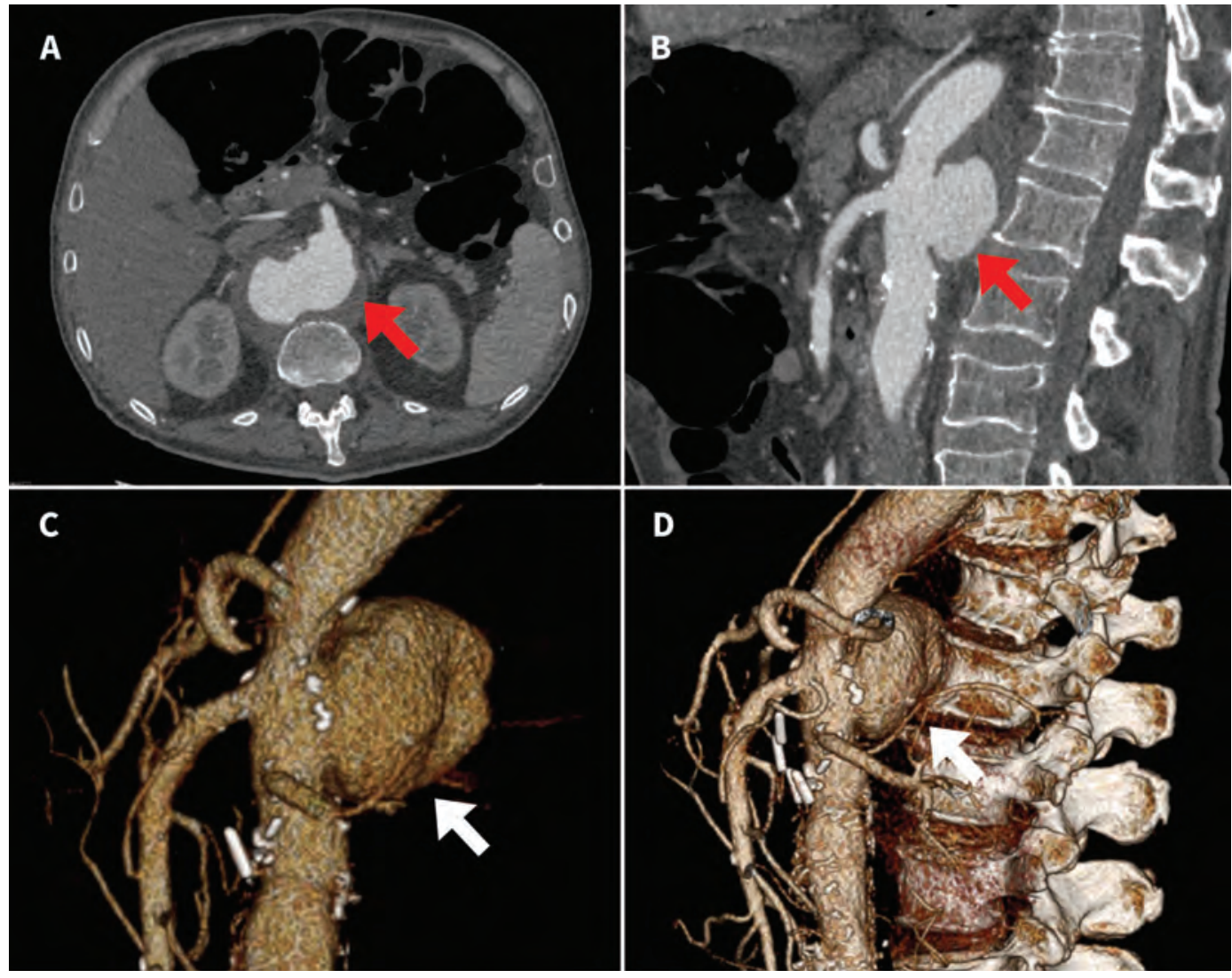

Figure 3: (A) Axial and (B) sagittal views on computed tomographic (CT) angiography performed before surgical intervention. A wide-mouth pseudoaneurysm (red arrows) involving the posterior wall of the abdominal aorta at the level of the celiac axis and superior mesenteric arteries $(6.7 \times 5.0 \times 3.7 \mathrm{~cm})$ can be seen. $(C$ and $D)$ Three-dimensional volume rendering of abdominal CT imaging data showing a wide-mouth pseudoaneurysm (white arrows).

systemic complications. Localized reactions, which occur in 30\%$60 \%$ of patients, include hematuria, lower urinary tract symptoms such as dysuria and frequency and, not infrequently, transient fever and malaise. ${ }^{3}$ The mechanism for systemic infection, which occurs in less than $5 \%$ of patients, is unknown and likely involves hematogenous spread from the instillation site promoted by local cystitis secondary to catheter-related trauma or biopsy. ${ }^{5}$

\section{Systemic disease after BCG therapy}

Systemic disease after BCG therapy is divided into early (within three months) and late (more than one year after initial instillation) onset. Early complications typically include pneumonitis and hepatitis; less than $30 \%$ of patients have positive cultures. ${ }^{6}$

Late disease manifests after several instillations, and the presentation is varied. ${ }^{6,7} \mathrm{New}$-onset back pain accompanied by general malaise, fever, night sweats and chills in patients undergoing BCG therapy should prompt consideration of vertebral osteomyelitis (Pott disease), with associated epidural abscesses in the differential diagnosis. There are at least seven reported cases of vertebral osteomyelitis after BCG therapy, typically involving men more than 65 years of age. ${ }^{8}$

In our patient's case, initial screening of lumbar vertebrae by MRI with gadolinium enhancement did not show osteomyelitis or discitis. At that point (before CT angiography), the consensus among the medical team was to investigate for alternative causes of our patient's decline, including recurrent or new malignant disease or bony disease from an alternative process, such as mechanical musculoskeletal causes.

\section{Mycotic aneurysm}

Almost 30 cases of mycotic aneurysms after BCG therapy have been reported, mostly involving the thoracic or abdominal aorta, although some have involved coronary arteries. ${ }^{9-11}$ Similar to bony disease, mycotic aneurysm can present with back pain, fever, chills and general malaise. The current imaging method of choice for detecting mycotic aneurysm is multidetector CT angiography, which provides images quickly and with high sensitivity. ${ }^{12}$ Magnetic resonance imaging is a reasonable alternative; however, studies that focus on discitis or osteomyelitis may miss aneurysm formation, as in our patient's case.

An analysis of previous cases by Holmes and colleagues highlights how the nonspecific presentation of mycotic aneurysm often accounts for delays in diagnosis. ${ }^{10}$ Previous reports suggest a mean time to diagnosis for mycotic aneurysm formation of 23 months after the most recent instillation of BCG. This result was further emphasized by a study that involved 43 patients who had infected aneurysms; the most common set of clinical and laboratory features found on presentation (> 50\%) included insidious nonspecific symptoms, elevations in erythrocyte sedimentation rates and leukocytosis. ${ }^{7}$ 
Previous case reports of ruptured mycotic aneurysm after BCG therapy have raised the consideration for screening for aneurysms before BCG immunotherapy is started and, if an aneurysm is found on screening, whether patients should undergo the treatment. ${ }^{6}$ The current guideline from the Canadian Urological Association recommends that patients with high risk for nonmuscle invasive bladder cancer undergo CT urography every one to two years (grade $\mathrm{C}$ recommendation). ${ }^{1}$ However, to our knowledge, no specific guideline exists that recommends patients with bladder cancer undergo additional screening for aneursyms, beyond the recommendations in the recent Canadian Task Force on Preventive Health Care guideline on screening for abdominal aortic aneurysm. ${ }^{13}$

Arterial injury from atherosclerotic plaques, pre-existing aneurysm or iatrogenic mechanism related to vascular access coupled with impaired immunity should raise clinical suspicion for aneurysm formation. The most common risk factors for atherosclerotic aneurysm formation are type 2 diabetes, hypertension and hyperlipidemia, and patients with these conditions may also have an increased risk for secondary infection. ${ }^{14}$ Infected aneurysms may also arise via contiguous spread of BCG from local tissue including vertebral bodies and, as previously stated, should be ruled out as part of the underlying cause.

\section{Kidney disease}

A unique aspect of our patient's case is renal disease as part of the manifestation of disseminated disease. Previous reports have described granulomatous, interstitial or hypocomplementemic kidney disease with BCG therapy. ${ }^{15}$ Our patient had interstitial nephritis with noncaseating granulomas (Figure 2), which is an uncommon complication of BCG therapy. Renal failure has been reported to occur up to six months after BCG instillation. In our patient's case, we saw evidence of kidney injury within three months.

Mechanisms to explain renal involvement are unclear, and most cases of renal injury related to BCG instillation are related to sepsis and secondary to mycobacteremia or other genitourinary infections. ${ }^{15}$ In reviewing our patient's chart, we found no evidence of sepsis during his most recent transurethral resection surgery and instillation of BCG; no cultures were positive for blood or urine infection at the time of the initial kidney injury. The patient's renal function improved to near baseline with anti-tuberculous therapy.

\section{Conclusion}

Our patient's case shows the need for a high degree of suspicion of BCG-related systemic disease when patients present with new back pain, malaise and functional decline. Infection of an aortic aneurysm is uncommon, but should be considered when patients present with new back pain after having undergone BCG therapy. This scrutiny may be particularly important for patients with vascular risk factors who may be predisposed to aneurysm formation. The diagnostic work-up should include assessment for vertebral discitis or osteomyelitis as a distinct entity or as a source of local extension to the aorta.

Kidney function should be monitored for patients receiving recurrent instillations, and interstitial nephritis should be part of the differential diagnosis when patients present with either early or delayed acute kidney injury after having received intravesicular therapy.

\section{References}

1. Kassouf W, Traboulsi SL, Kulkarni GS, et al. CUA guidelines on the management of nonmuscle invasive bladder cancer. Can Urol Assoc J 2015;9:E690-704.

2. Chou R, Selph S, Buckley DI, et al. Intravesical therapy for the treatment of nonmuscle invasive bladder cancer: a systematic review and meta-analysis. J Urol 2017;197:1189-99.

3. Safdar N, Abad CL, Kaul DR, et al. Clinical problem-solving. An unintended consequence-a 79-year-old man with a 5-month history of fatigue and 20-lb (9-kg) weight loss presented to his local physician. N Engl J Med 2008;358:1496-501.

4. Kresowik TP, Griffith TS. Bacillus Calmette-Guérin immunotherapy for urothelial carcinoma of the bladder. Immunotherapy 2009;1:281-8.

5. To U, Kim J, Chia D. Disseminated BCG: complications of intravesical bladder cancer treatment. Case Rep Med 2014;2014:362845.

6. Gonzalez OY, Musher DM, Brar I, et al. Spectrum of bacille Calmette-Guérin (BCG) infection after intravesical BCG immunotherapy. Clin Infect Dis 2003;36:140-8.

7. Macleod LC, Ngo TC, Gonzalgo ML. Complications of intravesical Bacillus CalmetteGuérin. Can Urol Assoc J 2014;8:E540-4.

8. Josephson CB, Al-Azri S, Smyth DJ, et al. A case of Pott's disease with epidural abscess and probable cerebral tuberculoma following Bacillus Calmette-Guérin therapy for superficial bladder cancer. Can J Infect Dis Med Microbiol 2010;21:e75-8.

9. Holmes BJ, LaRue RW, Black JH III, et al. Mycotic aortic aneurysm due to intravesical BCG immunotherapy: clinical manifestations and diagnostic challenges. Int $\mathrm{J}$ Mycobacteriol 2014;3:60-5

10. Simar J, Belkhir L, Tombal B, et al. Ruptured aortic aneurysm due to Mycobacterium bovis $B C G$ with a delayed bacteriological diagnosis due to false negative result of the MPB 64 immunochromatographic assay. BMC Res Notes 2017;10:64.

11. Duvnjak P, Laguna M. Left anterior descending coronary artery and multiple peripheral mycotic aneurysms due to mycobacterium bovis following intravesical Bacillus Calmette-Guérin therapy: a case report. J Radiol Case Rep 2016;10:12-27.

12. Lee WK, Mossop PJ, Little AF, et al. Infected (mycotic) aneurysms: spectrum of imaging appearances and management. Radiographics, 2008;28:1853-68.

13. Canadian Task Force on Preventive Health Care. Recommendations on screening for abdominal aortic aneurysm in primary care. CMAJ 2017;189:E1137-45.

14. Oderich GS, Panneton JM, Bower TC, et al. Infected aortic aneurysms: aggressive presentation, complicated early outcome, but durable results. J Vasc Surg 2001;34:900-8.

15. Manzanera Escribano MJ, Morales Ruiz E, Odriozola Grijalba M, et al. Acute renal failure due to interstitial nephritis after intravesical instillation of BCG. Clin Exp Nephrol 2007;11:238-40.

\section{Competing interests: None declared.}

This article has been peer reviewed.

The authors have obtained patient consent.

Affiliations: Postgraduate Medical Education, Department of Medicine (Rohailla), University of Toronto; Division of Nephrology, Department of Medicine (Kitchlu); Division of Vacsular
Surgery, Department of Surgery (Wheatcroft); and Division of General Internal Medicine, Department of Medicine (Razak), St. Michael's Hospital, University of Toronto, Toronto, Ont.

Contributors: Sagar Rohailla and Fahad Razak wrote the manuscript. Sagar Rohailla, Abhijat Kitchlu, Mark Wheatcroft and Fahad Razak edited the manuscript for important intellectual content and approved the final version to be published.

Acknowledgement: The authors thank Dr. Robert J. Stewart for his assistance in the technical editing of the manuscript.

Correspondence to: Fahad Razak, fahad.razak@mail.utoronto.ca 